Anat. Embryol. 148, 333 (1975)

(C) by Springer-Verlag 1975

\title{
Erratum
}

\section{A Geometric Model of the Rat Kidney}

R. Kainer

Anat. Embryol. 147, 91-109 (1975)

The formula $5 \mathrm{c}$ on page 93 must read:

$$
d a=\gamma_{P}^{(N-2)} \frac{s \sqrt{N}}{\sqrt{\bar{N}-2}} \sqrt{\frac{\sum_{i=1}^{n} g_{i} m_{i} x_{i}^{2} / \sum_{i=1}^{n} g_{i} m_{i}}{\sum_{i=1}^{n} g_{i} m_{i}\left(x_{i}-\bar{x}\right)^{2}}}=d b \sqrt{\overline{x^{2}}}
$$

Dipl.-Ing. R. Kainer Institut für Systemdynamik und Regelungstechnik Universität Stuttgart Keplerstraße 17 D-7000 Stuttgart 1 Federal Republic of Germany 